$\underline{\beta}=-1$

\title{
Assessment of selected heavy metal load and identification of bacterial status associated with suya meat sold in Anambra state, Nigeria
}

\author{
R. N. Asomugha ${ }^{1}$, E. E. Ilodigwe ${ }^{1}$, J. O. Amedu ${ }^{1}$, M. Ogbonnaya ${ }^{1}$, E. Ifediba ${ }^{2}$ \\ ${ }^{1}$ Department of Pharmacology and Toxicology, Faculty of Pharmaceutical Sciences, Nnamdi Azikiwe University, Awka, Nigeria \\ ${ }^{2}$ Department of Pharmacology and Therapeutics, Faculty of Medicine, Nnamdi Azikiwe University, Nnewi Campus \\ *Corresponding author E-mail: rn.asomugha@unizik.edu.ng
}

\begin{abstract}
Heavy metals and some pathogenic bacteria tend to contaminate food substances commonly consumed and this pose as health risks to the populace. The study was therefore conducted to assess the level of metal and bacteria contamination of meat barbecue (SUYA) commonly consumed in Anambra State, Nigeria. Thirty six sample were evenly collected from the sensational Zones and analyzed using Atomic Absorption Spectrophotometer. Metal values obtained range as follows: Cadmium(4.17-7.93mg/kg), Lead(0.24-3.39mg/kg),Zinc(2.6$28.3 \mathrm{mg} / \mathrm{kg}$ ) and Arsenic $(0.19-2.3 \mathrm{mg} / \mathrm{kg}$ ). Samples from Anambra North had the highest Zinc, Lead, and Arsenic levels 14.02, 0.94, and $0.96 \mathrm{mg} / \mathrm{kg}$ rewspectively, while Anambra South recorded the highest cadmium level $6.16 \mathrm{mg} / \mathrm{kg}$. The Estimated daily intake(EDI) of Cadmium alone was found to be greater than the recommended value in the 3 senatorial zones. Again only Cadmium had a target hazard quotient(THQ) of greater than one in all the senatorial zones. All the zones had hazard index (HI) greater than one. North(5.95+00). South, $(8.1 \mathrm{E}+00)$ and Central(4.8+00). Cancer risk for cadmium recorded in all the zones were above accepted limits. While for Arsenic the North zone recorded levels above acceptable limits. The following bacteria species were isolated from analyze soya samples. Staphyloccocus cereus, Bacillus aureus, Salmonella species, Pseudomonas aeruginosa, Klebsiella pneumonia,however bacillus cereus was most commonly isolated and Pseudomonas aeruginosa least commonly isolated. The results indicated an appreciable level of contamination and a possible public health risk.
\end{abstract}

Keywords: Heavy Metals; Bacterial Load; Suya; Cancer Risk.

\section{Introduction}

Heavy metals and various pathogenic bacteria are most dangerous among the pollutants of urbanization, because of serious health problems it tends to cause to human population (Ojebah et al., 2015). As a consequence of natural and anthropogenic activities, heavy metals are present in the environment, so that people come into contact with them especially through the consumption of foods (Hărmanescu et al., 2011).

Suya is a traditional beef barbecue, smoked or roasted, obtained from thinly sliced boneless meat and marinated with various spices such as clove, ginger, pepper, salt, peanut cake, vegetable oil as well as food additives and flavorings (Akpamu 2011; Egbebi and Seidu, 2011), enjoyed as a delicacy in West Africa (Eke et al., 2013). It is a street-vended food which provides a source of inexpensive, convenient and often nutritious menu for cities, urban and rural areas. It serves as a major source of income for vast number of persons, and creates opportunity for self-employment (Omojola, 2008).

However, the preparation of food usually result in their inadvertent bacterial contamination (Mead, 2004) especially if improperly handled or abused. Some of these genera are known to be of public health concern and have been associated with cases of gastroenteritis and other food borne diseases (Moshood et al., 2012). The sources of these contaminations have been linked to poor hygienic conditions of the handlers and environment, raw meat, spices and packaging materials (Pavyde et al., 2015).

The fact that there are sporadic cases of gastroenteritis and symptoms of food infection after consumption of suya indicates that the products indeed constitute a food safety risk (Inyang et al., 2005; Odey et al., 2013). The main threats to human health are contamination with heavy metals, especially lead, cadmium and arsenic. Heavy metals become toxic when they are not metabolized by the body and accumulate in tissues (World Health Organization, 2000). They are dangerous because they tend to accumulate in living organisms. Some are deposited as residues in food, during processing (World Health Organization, 2011).

Furthermore, the most important bacterial meat spoilage is caused by lactic acid bacteria; these include many species such as Lactobacillus, Leuconostoc, Pediococcus and Streptococcus which are physiologically related to a group of fastidious and ubiquitous gram-positive organisms. The possible sources of contamination are through slaughtering of sick animals, washing the meat with dirty water by butchers, inappropriate exposure of the Suya meat, contamination by flies through processing done close to sewage or refuse dumps site, transportation by rickety vehicles, use of contaminated equipment such as, knife and other utensils and addition of unclean spices. 
Reports have shown that suya sold along the road are exposed to heavy metals emanating from automobiles (Innocent et al., 2017). Reports by several workers indicate that a wide spectrum of microbes such as Bacillus species, Clostridium species, Enterobacter species, Escherichia species, Klebsiella species, Micrococcus species, Proteus species, Pseudomonas species, Salmonella species, Shigella species, Staphylococcus species and Streptococcus species have been isolated from ready-to-eat suya in various parts of Nigeria (Uzeh et al., 2006; Moshood et al., 2012; Salihu et al., 2013).

The presence of heavy metals in the body as a result of excessive consumption from food at certain levels has dangerous effects and therefore calls for concern if allowed to accumulate. The excess heavy metals can cause deoxyribonucleic acid (DNA) damage and conformational changes that may lead to cell cycle modulation, carcinogenesis or apoptosis as a result of metal ion interaction with cell components such as DNA and nuclear proteins (Beyersmann et al., 2008). Previous research in Nigeria had identified high levels of heavy metals in suya meat. Dibofori et al (2018) found levels of cadmium and nickel to be above the acceptable limit in suya meat sold in Port Harcourt metropolis. Similarly, Ojebah et al (2015) found elevated level of cadmium in suya meat marketed in Delta state. However, there is no documented result from Anambra State, Nigeria thus, the need for this basic assessment of the metal load and bacterial status of Suya sold on the streets of Anambra State.

\section{Materials and methods}

\subsection{Study area}

Anambra state is one of the 36 states of Nigeria and is located in the South East geopolitical zone of Nigeria. Anambra states occupies a land mass of about 4, 844 square kilometer and is bounded in the East by Enugu state in the North by Kogi State , in the South by Rivers and Imo states and in the west by Delta state. The latest official 2006 census figures gave the population of the state as 4.06 million . the official projected population for the year 2020 is estimated at 6.18 million people. with a population density of 1270 people per sq. Kilometre (Chukwuma et al., 2016). The state is divided into 21 local government areas with Awka serving as its capital.

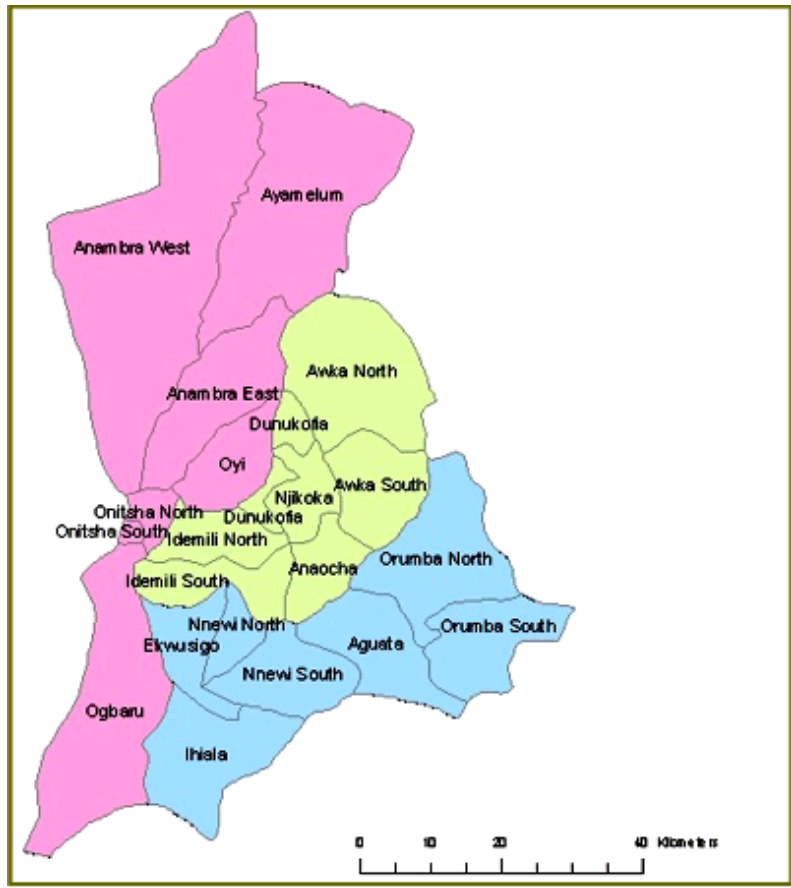

Fig. 3. 1: Diagram of the Map of Anambra State and Its Local Government (Chukwuma Et Al., 2016).

\subsection{Samples collection}

Thirty six (36) Suya samples were collected from the three senatorial zones, twelve (12) samples from each zone; Anambra North (Onitsha North, Onitsha South Ogbaru and Oyi LGAs), Anambra South (Nnewi North, Nnewi South, Ihiala and Ekwusigo) and Anambra Central (Njikoka, Awka South, Idemili South and Aniocha LGAs). All samples collected were wrapped in sterile aluminum foil to avoid further contamination and transported to the laboratory where they were analyzed for the presence of heavy metals and bacterial contamination.

The research was carried out at three different laboratories. Suya meat that served as control samples were prepared under hygienic and controlled environmental condition at the Pharmacology and Toxicology Laboratory, Nnamdi Azikiwe University Agulu Campus. Identification of the bacterial status in the suya meat was carried out in Microbiology laboratory, Agulu Campus of the Nnamdi Azikiwe University, and the heavy metal analysis of the suya meat was done in Biotechnology Laboratory, Nnamdi Azikiwe University Awka, Anambra State.

\subsection{Determination of microbial loads of suya samples}

Ten grams $(10 \mathrm{~g})$ of suya from each sample was removed from the skewers, and mashed in a sterile laboratory type mortar and pestle. Exactly $1 \mathrm{~g}$ of the mashed suya meat was weighed and then aseptically transferred into a corresponding sterile test tube containing $9 \mathrm{ml}$ of sterile distilled water, properly shaken and sieved before a tenfold dilution was performed. Using a pour plate technique, one milliliter ( $1 \mathrm{ml})$ aliquot of dilution were transferred to clean sterilized petri dishes mixed with $20 \mathrm{ml}$ of sterile molten Nutrient Agar, Centrimide Agar, Salmonella and Shigella Agar, Mannitol salt Agar, MacConkey Agar for bacteria and Saubouraud Dextrose Agar for fungi which 
was cooled to $45^{\circ} \mathrm{C}$ for bacteria and fungi respectively. The plates were allowed to set and then incubated at $37^{0} \mathrm{C}$ for bacterial counts from $18-24$ hrs and $27^{\circ} \mathrm{C}$ for fungi count for $72-168$ hours. All counts were expressed in CFU/ml. Pure cultures were obtained from the plates, stored on agar slants and kept in the refrigerator until used for characterization of samples.

Characterization of microorganisms isolated from Suya samples

Microorganisms growing on solid surface medium tend to form colonies with distinctive morphologies. The variations in bacterial colony morphology can be observed with naked eye. These morphological variations include shape, odour, and elevation surface on agar medium. The bacteria isolates were also characterized by their gram staining reactions (microscopy) and confirmatory biochemical tests.

\subsection{Gram staining technique}

Smears of the isolates were made on clean grease - free slides, air dried and heat fixed. These were covered with giemsa stain for 60 seconds and rinsed with distilled water. Lugol's iodine was then added for 60 seconds and rinsed with distilled water. They were then rapidly decolorized for 8 seconds with alcohol and immediately rinsed with distilled water. They were counter stained with Safranin red for 2 minutes and rinsed with clean water. The back of the slides were wiped dry and examined under the oil immersion microscope for Gram characteristics of the organisms (Cheesbrough, 2009).

\subsection{Biochemical tests}

The following biochemical tests were conducted to confirm the results obtained from the macroscopic and microscopic examination of the isolates; Indole, Coagulate, Oxidase, Citrate tests and starch hydrolysis (Cheesbrough, 2009), Catalase test (Reiner, 2010).

\subsection{Preparation of suya ingredients}

The spices that were used in preparing the control, Suya spices were purchased from Nkwo market Nnewi, Anambra state. They include ginger (Zingiberofficinales), clove (Syzygium aromaticum), garlic (Allium sativum), Cayenne pepper (Capsicum annuum) flakes and African Negro pepper (Annonaceae) commonly known as Uda in Igbo. Other constituents of the ingredients were groundnut cake powder (Arachis hypogea) salt and other seasonings.

The spices and other ingredient constituents were milled individually and mixed together in a specific proportion as described by Omojola (2008) since the ingredients used by the processors of suya in Anambra State are similar to that used by the author. All the other processing steps were followed.

\subsection{Preparation of samples for metal analysis}

Ten grams each of the suya samples from each group were weighed out using an analytical balance (Helmenstine, 2017) and poured into a digestion beaker. A $100 \mathrm{ml}$ volume of aqua-ragia $\left(70 \% \mathrm{HCl}+30 \% \mathrm{HNO}_{3}\right)$ was poured into the beaker and heated in a Khedjal apparatus heating mantle placed in a fume cupboard. The digestion continued until the volume of the acid was reduced and a clear and colorless solution had been attained. The digested sample was then filtered using filter paper and made up to $100 \mathrm{ml}$ with distilled water.

\subsection{Determination of heavy metals and method for analysis}

A series of standard metal solutions in the optimum concentration range was prepared by preparing from the standard stock solution (1000ppm buck standard solution). From the stock solution, we prepared standard solutions with the following concentrations: 60ppm, $30 \mathrm{ppm}$ and $10 \mathrm{ppm}$. The first one was made to be at the top of the linear range. The concentrations of the second standard were approximately 3 times the concentration of the first. The concentration of the third standard was approximately 6 times the concentration of the first standard (6:3:1 rule) (Potts et al., 2004). The AAS instrument in the calibration mode was auto zeroed using ionized water as blank. The first standard (max) was set as S1. The second and third standard was also calibrated to S2 and S3 (min) respectively. Calibration curve for each metal was prepared by plotting the absorbance of the standard versus their concentrations. The measurement was repeated three times.

\subsection{Determination of food ingestion rate (FIR)}

To determine the food ingestion rate $\left(\mathrm{F}_{\mathrm{IR}}\right)$, a survey analysis was conducted using a questionnaire (Appendix 1). The food frequency questionnaires were standardized to times per day with the following rules: for months, reported frequency was divided by 28 ; for week, reported frequency was divided by 7 and for day, frequency was as reported. For ranges like $2-3,2.5$ were used as the midpoint and this was multiplied by the average weight of suya (Winter, 2015). Thus,

$F_{I R}=$ average weight of suya $x$ average frequency of consumption per day

\subsection{Estimated daily intake of metal (EDI)}

The estimated daily intake (EDI) of metal ( $\mu \mathrm{g} / \mathrm{kg} / \mathrm{day})$ was calculated based on integration of data from analysis of heavy metals, meat ingestion rate (from survey), and body weight of Nigerian adults. The EDI for all the metals were obtained using the following equation described by the Human Health Evaluation Manual (USEPA, 2012).

Estimated Daily Intake $(E D I)=\frac{C \times \text { IngR x EFr x ED }}{B w \times \text { Atn }}$

$\mathrm{C}=$ concentration of heavy metal in suya delicacy, $\mathrm{IngR}=$ ingestion rate (Ingestion rate $(\mathrm{g} /$ day $)=$ Ingestion frequency $\left(\right.$ day $\left.{ }^{-1}\right) \times$ Portion size $($ gram $)), \mathrm{EFr}=$ exposure frequency $($ days/year $)=365, \mathrm{ED}=$ exposure duration (years), Atn= average time $=365 \mathrm{x} \mathrm{ED}$ 


\subsection{Non-carcinogenic risk}

\subsection{Target hazard quotient (THQ)}

Hazard quotient was determined from the following equation

Target Hazard Quotient $($ THQ $)=$ EDI x $10^{-3-}$

Rfd

Rfd $=$ Reference dose of metal (Table 2 in appendix)

\subsection{Hazard index $(\mathrm{HI})$}

Hazards index was generated using the equation:

$\mathrm{HI}=\Sigma \mathrm{HQi}$

Where i represents each metal analyzed.

A HQ and/or HI of $>1$ indicate that there is potential risk of the contaminant to human health, whereas a result of $\leq 1$ indicates no obvious risk of adverse health effects. This risk assessment method has been used by other researchers (Isa et al., 2015).

\subsection{Cancer risk (CR)}

The cancer risk of an individual was estimated by calculating the increasing chances to develop cancer over a life time due to exposure to a contaminant. This was achieved using cancer slope factor (SF) which converts the approximate daily intake of a toxic substance for exposure in a life time to the increasing risk of an individual to develop cancer (IARC, 2011).

Cancer risk was generated using the equation:

Cancer Risk $=$ Oral Slope Factor $\left[(\mathrm{mg} / \mathrm{kg} \cdot \mathrm{day})^{-1}\right] \mathrm{x}$ EDI

Where risk is the probability of an individual to develop cancer, EDI is the average daily intake in $\mu \mathrm{g} / \mathrm{kg} / \mathrm{day}, \mathrm{SF}$ is the carcinogenic slope factor $(\mathrm{mg} / \mathrm{kg} / \mathrm{day})^{-1}$. The USEPA considers acceptable for regulatory purposes a cancer risk in the range of $1 \mathrm{E}-06$ to $1 \mathrm{E}-04$.

Oral slope factor for $\mathrm{As}=1.5 \mathrm{E}+00$

Oral slope factor for $\mathrm{Pb}=8.5 \mathrm{E}-03$

Oral slope factor for $\mathrm{Cd}=6.3 \mathrm{E} 00$

(IARC, 2011).

\subsection{Statistical analysis}

Data obtained were analyzed using the Statistical Package for Social Sciences (SPSS) version 20, and results were expressed as median. Significant differences among the four groups were obtained using Kruskal-Wallis test (nonparametric), while pairwise comparison (post hoc) test was done using Bonferroni corrections.

\section{Results}

\subsection{Concentration of heavy metals in suya meat}

The levels of cadmium, lead, zinc and arsenic in suya from different zones in Anambra state were analyzed and the health risk assessment estimated. The result in appendix 1 and 2 shows the mean concentration of the various metals $(\mathrm{Pb}, \mathrm{Cd}, \mathrm{As}$ and $\mathrm{Zn})$ in suya meat sold in the street of Anambra state. The mean concentration $(\mathrm{mg} / \mathrm{kg})$ of the various metals in the suya meat samples ranged within (4.177.93) $\mathrm{Cd},(0.24-3.13) \mathrm{Pb},(2.6-23.8) \mathrm{Zn}$ and (0.19-2.3) As in $\mathrm{mg} / \mathrm{kg}$ respectively. The comparison among zones and control was analyzed using the non-parametric Kruskal- Wallis test. From Table 4.1, the levels of cadmium in suya meat samples from Anambra north, south, and central differed significantly $\mathrm{Cd}(\mathrm{p}=0.001)$ from the control sample but there were no significant difference for $\mathrm{Zn}(\mathrm{p}=0.288)$ and As $(p=0.166)$ and lead $(p=0.63)$ between the zones and the control. The mean concentration of heavy metals are shown in tables $4.1 \mathrm{a}$, $4.1 \mathrm{~b}$ and $4.1 \mathrm{c}$.

Table 4.1: A) Mean Concentration of Heavy Metals in Suya Samples in Anambra North and Control.

\begin{tabular}{llll}
\hline Area & Cadmium $(\mathrm{mg} / \mathrm{kg})$ & Lead $(\mathrm{mg} / \mathrm{kg})$ & Zinc $(\mathrm{mg} / \mathrm{kg})$ \\
\hline Onitsha north & $4.17 \pm 0.16$ & $1.23 \pm 0.03$ & $2.60 \pm 1.74$ \\
& $6.25 \pm 0.10$ & $1.84 \pm 0.03$ & $10.98 \pm 2.20$ \\
Onitsha south & $4.74 \pm 0.57$ & $1.18 \pm 0.03$ & $21.4 \pm 0.85$ \\
& $7.10 \pm 0.06$ & $3.13 \pm 0.55$ & $27.38 \pm 1.46$ \\
& $6.14 \pm 0.53$ & $1.50 \pm 0.02$ & $17.05 \pm 0.85$ \\
Ogbaru & $7.57 \pm 0.47$ & $2.66 \pm 0.22$ & $11.95 \pm 2.16$ \\
& $6.23 \pm 0.08$ & $0.70 \pm 0.03$ & $22.02 \pm 1.32$ \\
& $6.15 \pm 2.08$ & $0.67 \pm 0.03$ & $16.94 \pm 0.05$ \\
& $5.82 \pm 0.89$ & $0.52 \pm 0.04$ & $1.03 \pm 0.2 \pm 1.22$
\end{tabular}




\begin{tabular}{|c|c|c|c|c|}
\hline \multirow[t]{3}{*}{ Oyi } & $4.48 \pm 0.71$ & $0.38 \pm 0.03$ & $8.3 \pm 1.60$ & $0.24 \pm 0.05$ \\
\hline & $4.09 \pm 0.07$ & $0.22 \pm 0.05$ & $6.34 \pm 0.81$ & $0.27 \pm 0.01$ \\
\hline & $5.93 \pm 0.64$ & $0.26 \pm 0.03$ & $9.81 \pm 1.61$ & $0.22 \pm 0.01$ \\
\hline \multirow[t]{6}{*}{ Control } & $1.99 \pm 0.06$ & $0.43 \pm 0.08$ & $12.94 \pm 2.37$ & $0.27 \pm 0.03$ \\
\hline & $1.10 \pm 0.10$ & $0.43 \pm 0.08$ & $12.94 \pm 2.37$ & $0.29 \pm 0.04$ \\
\hline & $2.37 \pm 0.17$ & $0.34 \pm 0.05$ & $14.57 \pm 0.74$ & $0.11 \pm 0.01$ \\
\hline & $1.96 \pm 0.06$ & $0.31 \pm 0.01$ & $11.53 \pm 1.33$ & $0.29 \pm 0.04$ \\
\hline & $1.37 \pm 0.05$ & $0.16 \pm 0.01$ & $17.89 \pm 0.24$ & $0.04 \pm 0.02$ \\
\hline & $2.12 \pm 0.05$ & $0.22 \pm 0.02$ & $15.65 \pm 0.53$ & $0.18 \pm 0.02$ \\
\hline WHO & $0.5 \mathrm{mg} / \mathrm{kg}$ & $0.2 \mathrm{mg} / \mathrm{kg}$ & - & $0.1 \mathrm{mg} / \mathrm{kg}$ \\
\hline CODEX & $0.1 \mathrm{mg} / \mathrm{kg}$ & $0.4 \mathrm{mg} / \mathrm{kg}$ & $60 \mathrm{mg} / \mathrm{kg}$ & - \\
\hline
\end{tabular}

Values are presented as mean \pm Standard error of mean (SEM), $\mathrm{n}=3$

Key: WHO - World Health Organization (2011)

CODEX- Codex Alimentarius Commission (2011)

Table 4.1: B) Mean Concentration of Heavy Metals in Suya Samples in Anambra South and Control.

\begin{tabular}{|c|c|c|c|c|}
\hline Areas & Cadmium (mg/kg) & Lead (mg/kg) & Zinc $(\mathrm{mg} / \mathrm{kg})$ & Arsenic $(\mathrm{mg} / \mathrm{kg})$ \\
\hline \multirow[t]{3}{*}{ Ekwusigo } & $6.09 \pm 0.54$ & $1.31 \pm 1.01$ & $14.25 \pm 0.81$ & $0.24 \pm 0.04$ \\
\hline & $6.24 \pm 0.15$ & $0.06 \pm 0.03$ & $24.97 \pm 0.99$ & $0.27 \pm 0.03$ \\
\hline & $6.25 \pm 0.49$ & $0.58 \pm 1.00$ & $9.48 \pm 1.39$ & $0.75 \pm 0.57$ \\
\hline \multirow[t]{3}{*}{ Nnewi south } & $5.98 \pm 1.45$ & $0.58 \pm 1.00$ & $24.59 \pm 0.52$ & $1.29 \pm 0.02$ \\
\hline & $7.76 \pm 0.45$ & $0.84 \pm 0.05$ & $6.26 \pm 0.99$ & $1.73 \pm 0.02$ \\
\hline & $5.63 \pm 0.21$ & $1.15 \pm 0.03$ & $5.12 \pm 1.02$ & $0.23 \pm 0.06$ \\
\hline \multirow[t]{3}{*}{ Nnewi north } & $5.28 \pm 0.23$ & $3.20 \pm 0.27$ & $6.74 \pm 1.59$ & $2.29 \pm 0.02$ \\
\hline & $6.24 \pm 1.80$ & $0.24 \pm 0.05$ & $11.78 \pm 1.37$ & $0.19 \pm 0.02$ \\
\hline & $7.93 \pm 0.58$ & $0.62 \pm 0.04$ & $5.72 \pm 0.91$ & $0.25 \pm 0.05$ \\
\hline \multirow[t]{3}{*}{ Ihiala } & $5.38 \pm 0.16$ & $0.61 \pm 0.57$ & $2.83 \pm 0.35$ & $0.42 \pm 0.05$ \\
\hline & $4.26 \pm 0.31$ & $0.25 \pm 0.04$ & $13.83 \pm 0.86$ & $0.17 \pm 0.02$ \\
\hline & $7.54 \pm 0.53$ & $0.24 \pm 0.04$ & $8.89 \pm 0.59$ & $0.29 \pm 0.05$ \\
\hline \multirow[t]{6}{*}{ Control } & $1.99 \pm 0.06$ & $0.43 \pm 0.08$ & $12.94 \pm 2.37$ & $0.27 \pm 0.03$ \\
\hline & $1.10 \pm 0.10$ & $0.43 \pm 0.08$ & $12.94 \pm 2.37$ & $0.29 \pm 0.04$ \\
\hline & $2.37 \pm 0.17$ & $0.34 \pm 0.05$ & $14.57 \pm 0.74$ & $0.11 \pm 0.01$ \\
\hline & $1.96 \pm 0.06$ & $0.31 \pm 0.01$ & $11.53 \pm 1.33$ & $0.29 \pm 0.04$ \\
\hline & $1.37 \pm 0.05$ & $0.16 \pm 0.01$ & $17.89 \pm 0.24$ & $0.40 \pm 0.02$ \\
\hline & $2.21 \pm 0.05$ & $0.22 \pm 0.02$ & $15.65 \pm 0.53$ & $0.18 \pm 0.02$ \\
\hline WHO & $0.5 \mathrm{mg} / \mathrm{kg}$ & $0.2 \mathrm{mg} / \mathrm{kg}$ & - & $0.1 \mathrm{mg} / \mathrm{kg}$ \\
\hline CODEX & $0.1 \mathrm{mg} / \mathrm{kg}$ & $0.4 \mathrm{mg} / \mathrm{kg}$ & $60 \mathrm{mg} / \mathrm{kg}$ & - \\
\hline
\end{tabular}

Values are presented as mean \pm Standard error of mean $(\mathrm{SEM}), \mathrm{n}=3$

Key: WHO- World Health Organization (2011)

CODEX - Codex Alimentarius Commission (2012)

Table 4.1: C) Mean Concentration of Heavy Metals in Suya Samples in Anambra Central and Control

\begin{tabular}{|c|c|c|c|c|}
\hline Areas & Cadmium (mg/kg) & Lead $(\mathrm{mg} / \mathrm{kg})$ & Zinc $(\mathrm{mg} / \mathrm{kg})$ & Arsenic ( $\mathrm{mg} / \mathrm{kg})$ \\
\hline \multirow[t]{3}{*}{ Awka south } & $6.46 \pm 0.68$ & $2.33 \pm 1.00$ & $6.83 \pm 1.68$ & $0.59 \pm 0.71$ \\
\hline & $6.75 \pm 0.42$ & $1.94 \pm 0.56$ & $16.97 \pm 2.04$ & $0.57 \pm 0.01$ \\
\hline & $5.69 \pm 1.03$ & $0.16 \pm 0.02$ & $16.2 \pm 1.22$ & $0.15 \pm 0.01$ \\
\hline \multirow[t]{3}{*}{ Anaocha } & $5.94 \pm 0.32$ & $1.78 \pm 0.59$ & $11.46 \pm 1.13$ & $1.09 \pm 0.59$ \\
\hline & $5.32 \pm 0.33$ & $0.49 \pm 0.02$ & $13.65 \pm 4.02$ & $0.23 \pm 0.02$ \\
\hline & $5.60 \pm 0.53$ & $0.71 \pm 0.55$ & $5.25 \pm 0.07$ & $0.21 \pm 0.02$ \\
\hline \multirow[t]{3}{*}{ Idemili south } & $6.03 \pm 0.80$ & $0.87 \pm 0.66$ & $7.56 \pm 1.07$ & $0.43 \pm 0.09$ \\
\hline & $5.43 \pm 0.06$ & $0.45 \pm 0.08$ & $23.89 \pm 0.63$ & $0.20 \pm 0.02$ \\
\hline & $6.38 \pm 0.31$ & $0.68 \pm 0.04$ & $3.91 \pm 1.19$ & $0.33 \pm 0.01$ \\
\hline \multirow[t]{3}{*}{ Njikoka } & $5.86 \pm 1.59$ & $0.48 \pm 0.01$ & $12.21 \pm 0.73$ & $0.25 \pm 0.06$ \\
\hline & $5.58 \pm 0.04$ & $0.28 \pm 0.02$ & $7.22 \pm 1.63$ & $0.27 \pm 0.04$ \\
\hline & $5.75 \pm 0.35$ & $0.87 \pm 0.07$ & $5.29 \pm 1.29$ & $0.40 \pm 0.01$ \\
\hline \multirow[t]{6}{*}{ Control } & $1.99 \pm 0.06$ & $0.43 \pm 0.08$ & $12.94 \pm 2.37$ & $0.27 \pm 0.03$ \\
\hline & $1.10 \pm 0.10$ & $0.43 \pm 0.08$ & $12.94 \pm 2.37$ & $0.29 \pm 0.04$ \\
\hline & $2.37 \pm 0.17$ & $0.34 \pm 0.05$ & $14.57 \pm 0.74$ & $0.11 \pm 0.01$ \\
\hline & $1.96 \pm 0.06$ & $0.31 \pm 0.01$ & $11.53 \pm 1.33$ & $0.29 \pm 0.04$ \\
\hline & $1.37 \pm 0.05$ & $0.16 \pm 0.01$ & $17.89 \pm 0.24$ & $0.40 \pm 0.02$ \\
\hline & $2.12 \pm 0.05$ & $0.22 \pm 0.02$ & $15.65 \pm 0.53$ & $0.18 \pm 0.02$ \\
\hline WHO & $0.5 \mathrm{mg} / \mathrm{kg}$ & $0.2 \mathrm{mg} / \mathrm{kg}$ & - & $0.1 \mathrm{mg} / \mathrm{kg}$ \\
\hline CODEX & $0.1 \mathrm{mg} / \mathrm{kg}$ & $0.4 \mathrm{mg} / \mathrm{kg}$ & $60 \mathrm{mg} / \mathrm{kg}$ & - \\
\hline
\end{tabular}

Values are presented as mean as \pm Standard error of mean (SEM), $\mathrm{n}=3$

Key: WHO- World Health Organization ( 2011)

CODEX- Codex Alimentarius Commission (2012)

Table 4.2: Median Comparison of Metals across Zones and Control

\begin{tabular}{llll}
\hline Zones & Cadmium $(\mathrm{mg} / \mathrm{kg})$ & Lead $(\mathrm{mg} / \mathrm{kg})$ & Zinc $(\mathrm{mg} / \mathrm{kg})$ \\
\hline Ananmbra North & $6.03^{*}$ & 0.94 & 14.02 \\
Anambra South & $6.16^{*}$ & 0.61 & 9.19 \\
Anambra Central & $5.80^{*}$ & 0.69 & 9.96 \\
Combination of zones sampled & $5.84^{*}$ & 0.61 & 0.23 \\
Kruskal- Wallis test (p- value) & 0.001 & 0.063 & 0.30 \\
\hline
\end{tabular}

Values are presented using Kruskal- Wallis test, $* \mathrm{P}=0.001$. Significantly different from control.

\subsection{Estimated daily intake of selected metals $(\mathrm{mg} / \mathrm{kg} / \mathrm{day})$ in Suya meat}


Table 4.2 shows the estimated daily intake (EDI) of cadmium, lead, zinc and arsenic for an adult with a body weight of 70kg. The EDI values for cadmium, lead, zinc and arsenic in the suya samples from Anambra central, were 4.00, 0.48, 6.56 and 0.21 respectively while that of Anambra south were 4.49, 0.45, 6.71 and 0.20, respectively, Anambra north values were $3.77,0.58,8.77$ and 0.60 respectively. The values for combination of all the zones sampled were $3.98,0.41,6.43$ and 0.19 respectively.

Table 4.3: Estimated Daily Intake $\left(\mu \mathrm{gkg}^{-1} \mathrm{Day}^{-1}\right)$ Of Metals In Suya Processed In Different Zones

\begin{tabular}{|c|c|c|c|c|}
\hline \multirow{2}{*}{ Zones } & \multicolumn{4}{|c|}{ Metals (in $\mu \mathrm{g} / \mathrm{kg} /$ day) } \\
\hline & Cadmium & Lead & Zinc & Arsenic \\
\hline Anambra Central & 4.00 & 0.48 & 6.56 & 0.21 \\
\hline Anambra South & 4.49 & 0.45 & 6.71 & 0.20 \\
\hline Anambra North & 3.77 & 0.58 & 8.77 & 0.60 \\
\hline Mean concentration of all the zones sampled & 3.98 & 0.41 & 6.43 & 0.19 \\
\hline
\end{tabular}

\subsection{Health risk assessment}

The risk assessment (Non-carcinogenic risk and carcinogenic risk) results are presented in Tables 4.3a and b. From the table, the noncarcinogenic risk represents the Target hazard quotient (THQ) and Hazard index (HI). Target hazard quotient (THQ) of Cadmium in all the zones had values greater than 1 while the THQ of Lead, Zinc and Arsenic were <1 (Table 4.3a).

Results of the hazard indices (HI) are shown in Table 4.3a. All the zones; Anambra central (4.84E+00), Anambra south (8.10E+00), Anambra north (5.95E+00) and combination of all the zones sampled (4.74E+00) have HI greater than one. Furthermore, from the table, the highest $\mathrm{HI}$ of $8.10 \mathrm{E}+00$ was noted in samples from Anambra south while the lowest $\mathrm{HI}$ of 4.74E+00 was noted in mean for Anambra State.

Cancer risk values are presented in Table 4.3b. The USEPA considers acceptable for regulatory purposes a cancer risk in the range of 1.E-06 to 1.E-04 (This means that any value $>10^{-4}(1 . \mathrm{E}-04)$ is unacceptable risk). From the Table, the cancer risk for lead $(\mathrm{Pb})$ for all the zones (Anambra central, south, north and general) falls within the USEPA acceptable limit. Cancer risk for arsenic (As) for all the zones and combination of all the zones falls within the USEPA acceptable limit except in Anambra north while cancer risk for cadmium (Cd) for all the zones ( Anambra south, north, central) and general were higher than the acceptable limit.

Table 4.4: A) Non Carcinogenic Risk Assessment Of Metals In Suya Processed In Different Zones In Anambra State. (Target Hazard Quotient) And Hazard Index (HI)

\begin{tabular}{lllll}
\hline Zones & Cadmium & Lead & Zinc & Arsenic \\
\hline Central & $4.00 \mathrm{E} 00^{*}$ & $1.2 \mathrm{E}-01$ & $2.18 \mathrm{E}-02$ & $7.00 \mathrm{E}-01$ \\
South & $4.49 \mathrm{E} 00^{*}$ & $1.12 \mathrm{E}-01$ & $2.24 \mathrm{E}-02$ & $6.67 \mathrm{E}-01$ \\
North & $3.77 \mathrm{E} 00^{*}$ & $1.47 \mathrm{E}-01$ & $2.92 \mathrm{E}-02$ & $8.10 \mathrm{E} 00^{*}$ \\
Mean of zones sampled & $3.98 \mathrm{E} 00^{*}$ & $1.05 \mathrm{E}-01$ & $2.68 \mathrm{E}-02$ & $2.00 \mathrm{E} 00^{*}$ \\
\hline
\end{tabular}

*Indicates samples that pose non carcinogenic threat at values exceeding THQ $<1$ and $\mathrm{HI}<1$

Table 4.4: B) Carcinogenic Risk Assessment Of Metals In Suya Processed In Different Zones In Anambra State

\begin{tabular}{|c|c|c|c|}
\hline Zones & Lead & Arsenic & Cadmium \\
\hline Central & $4.08 \mathrm{E}-06$ & $3.15 \mathrm{E}-04$ & $2.52 \mathrm{E}-02 *$ \\
\hline South & $3.83 \mathrm{E}-06$ & $3.00 \mathrm{E}-04$ & $2.82 \mathrm{E}-02 *$ \\
\hline North & $5.01 \mathrm{E}-06$ & $9.00 \mathrm{E}-03^{*}$ & $3.77 \mathrm{E}-02 *$ \\
\hline Combination of zones sampled. & $3.57 \mathrm{E}-06$ & $2.85 \mathrm{E}-04$ & $2.50 \mathrm{E}-02 *$ \\
\hline
\end{tabular}

*Indicate samples that pose cancer threat considering cancer risk at values exceeding $1 \times 10^{4}(1 . \mathrm{E}-04)$ and values within range of $1 \times 10^{6}-1 \times 10^{4}(1 . \mathrm{E}-6-$ 1.E-04) as generally acceptable.

\subsection{Total viable bacterial count}

The total bacterial count, varied among Suya samples collected from various locations in Anambra. The least bacteria count was recorded at Awka south in Anambra central with $2.5 \times 10^{4} \mathrm{cfu} / \mathrm{ml}$ and no growth was found in the control sample as shown in Table $4.5 \mathrm{a}$ and $\mathrm{b}$.

\subsection{Occurrence and percentage distribution of isolated bacteria}

The occurrence and percentage distribution of the isolated bacteria are presented in Table 4.6 and 4.7. A total number of 71 bacteria species were isolated from the suya samples. Six different bacteria genera were isolated. The bacteria isolated were Escherichia coli, Staphylococcus auerus, Bacillus cereus, Salmonella species, Pseudomonas aeruginosa and Klebsiella aerogenes. Bacillus cereus was the most commonly occurring bacteria isolated with the percentage distribution of $31 \%$ (22) followed by Klebsiella pneumonia18\% (14) and Staphylococcus auerus $18 \%$ (14). Pseudomonas aeruginosa and Salmonella species had the least occurrence with $4 \%$ (3) and $10 \%$ (7) respectively. Shigella species was not detected in any of the suya samples.

Table 4.5: A) Total Viable Bacterial Count Of The Suya Samples Analyzed

\begin{tabular}{lll}
\hline SAMPLE SITE & TOTAL BACTERIA COUNT $10^{4} \mathrm{cfu} / \mathrm{g}$ & TOTAL FUNGI COUNT $10^{4} \mathrm{cfu} / \mathrm{g}$ \\
\hline AST I & 57 & 12 \\
AST2 & 25 & 35 \\
AST3 & 85 & 25 \\
AN1 & 91 & 41 \\
AN2 & 83 & 85 \\
AN3 & 49 & 17 \\
NNT 1 & TNTC & 7 \\
NNT2 & TNTC & 2 \\
NNT3 & 224 & 3 \\
NST 1 & TNTC & 3 \\
NST2 & TNTC & 7 \\
NST3 & 243 & 3 \\
IST 1 & TNTC & 7
\end{tabular}




\begin{tabular}{lll} 
IST 2 & TNTC & 5 \\
IST 3 & 118 & 5 \\
EKW 1 & TNTC & 3 \\
EKW 2 & TNTC & 13 \\
EKW 3 & 100 & 2 \\
IH 1 & TNTC & 2 \\
\hline Key: AS= Awka south, AN= Aniocha NNT= Nnewi north, NST= Nnewi south, IST= Idemili south, EKW= Ekwesigo, IH= Ihiala, TNTC= Too numerous \\
to count.
\end{tabular}

Table 4.5: B) Total Viable Bacterial Count Of The Suya Samples Analyzed

\begin{tabular}{|c|c|c|}
\hline Sample Site & Total Bacteria Count $10^{4} \mathrm{Cfu} / \mathrm{G}$ & Total Fungi Count $10^{4} \mathrm{Cfu} / \mathrm{G}$ \\
\hline $\mathrm{IH} 2$ & TNTC & 1 \\
\hline IH 3 & TNTC & 2 \\
\hline NJK 1 & 27 & 3 \\
\hline NJK 2 & 85 & 4 \\
\hline NJK 3 & 120 & NG \\
\hline OGB 1 & TNTC & 10 \\
\hline OGB 2 & TNTC & 15 \\
\hline OGB 3 & TNTC & 9 \\
\hline ONT 1 & TNTC & 17 \\
\hline ONT 2 & 241 & 5 \\
\hline ONT3 & TNTN & 13 \\
\hline OST 1 & TNTC & 27 \\
\hline OST 2 & TNTC & 20 \\
\hline OST 3 & 120 & 6 \\
\hline OYI 1 & 120 & 7 \\
\hline OYI 2 & 85 & 14 \\
\hline OYI 3 & 101 & 15 \\
\hline CONTROL & NG & NG \\
\hline
\end{tabular}

Key: $\mathrm{IH}=$ Ihiala, $\mathrm{NJK}=$ Njikoka, OGB$=$ Ogbaru, ONT $=$ Onitsha north, OST $=$ Onitsha south, NG= No growth, TNTC $=$ Too numerous to count .

Table 4.6: Frequency Of Isolation Of The Bacterial Isolates From The Suya Samples

\begin{tabular}{|c|c|c|c|c|c|c|c|}
\hline Sample site & Bacillus cereus & E.coli & K. Pneaumonia & P. Aeruginosa & Salmonella & S. auerus & Shigella \\
\hline AST & 2 & 1 & ND & ND & ND & ND & ND \\
\hline AN & 2 & 2 & 1 & ND & ND & 2 & ND \\
\hline NNT & 1 & ND & 2 & 1 & ND & ND & ND \\
\hline NST & 2 & 1 & 2 & ND & ND & 2 & ND \\
\hline IST & 3 & 1 & 2 & 1 & ND & ND & ND \\
\hline EKW & 3 & 1 & 1 & ND & ND & 3 & ND \\
\hline $\mathrm{IH}$ & 3 & 1 & 1 & ND & 1 & 2 & ND \\
\hline NJK & 1 & ND & 1 & ND & ND & 3 & ND \\
\hline ONT & ND & ND & 2 & ND & 2 & ND & ND \\
\hline OST & 1 & ND & 1 & 1 & 2 & 1 & ND \\
\hline OYI & 1 & 1 & 1 & ND & 1 & ND & ND \\
\hline
\end{tabular}

Key: AS= Awka south, AN= Aniocha NNT= Nnewi north, NST= Nnewi south, IST= Idemili south, EKW= Ekwesigo, IH= Ihiala, NJK= Njikoka, OGB= Ogbaru, ONT= Onitsha north, OST= Onitsha south, ND= Not Detected, E. coli= Escheria coli, K. Pneumonia = Klebsiella Pneumonia, P. Aeruginosa, S. Aureus $=$ Staphylococcus Aureus.

Table 4.7: Frequency and percentage distribution of the isolated bacteria

\begin{tabular}{lll}
\hline BACTERIAL ISOLATES & FREQUENCY & \% DISTRIBUTION \\
\hline Bacillus spp & 22 & 31 \\
E.coli & 11 & 15 \\
K. pneumonia & 14 & 18 \\
P.aeuroginosa & 3 & 4 \\
S.aureus & 14 & 18 \\
Salmonella & 7 & 10 \\
Shigella & ND & 0 \\
\hline ND= Not detected & & \\
\hline
\end{tabular}

$\mathrm{ND}=$ Not detected.

Table 4.8: A) Biochemical and Characterization Of Bacterial Isolates From Suya Meat

\begin{tabular}{|c|c|c|c|c|c|c|c|c|c|c|}
\hline Sample site & $\begin{array}{l}\text { Sample } \\
\text { code }\end{array}$ & $\begin{array}{l}\text { Grams } \\
\text { reaction }\end{array}$ & $\begin{array}{l}\text { Cellular } \\
\text { morphology }\end{array}$ & Catalase & Coagulase & Indole & Citrate & Oxidase & $\begin{array}{l}\text { Starch } \\
\text { hydrolysis }\end{array}$ & Probable identity \\
\hline \multirow[t]{3}{*}{ AST } & 1 & -ve & Rods & $+\mathrm{ve}$ & -ve & $+\mathrm{ve}$ & -ve & -ve & $-\mathrm{ve}$ & E.coli \\
\hline & 2 & + ve & Rods & $+\mathrm{ve}$ & -ve & -ve & $+\mathrm{ve}$ & -ve & $+\mathrm{ve}$ & Bacillus cereus \\
\hline & 3 & $+v e$ & Rods & $+\mathrm{ve}$ & -ve & -ve & $+\mathrm{ve}$ & -ve & $+\mathrm{ve}$ & Bacillus cereus \\
\hline \multirow[t]{7}{*}{ AN } & 1 & -ve & Rods & -ve & -ve & $+\mathrm{ve}$ & -ve & -ve & -ve & E.coli \\
\hline & & $+\mathrm{ve}$ & Rods & $+\mathrm{ve}$ & -ve & -ve & $+\mathrm{ve}$ & -ve & $+\mathrm{ve}$ & Bacillu cereus \\
\hline & & $+v e$ & Rods & $+\mathrm{ve}$ & $+\mathrm{ve}$ & -ve & $+\mathrm{ve}$ & -ve & -ve & Staph. Auruu s \\
\hline & 2 & + ve & Rods & $+\mathrm{ve}$ & -ve & -ve & $+\mathrm{ve}$ & -ve & + ve & Bacillu cereus \\
\hline & & -ve & Rods & -ve & -ve & $+\mathrm{ve}$ & -ve & -ve & -ve & E.coli \\
\hline & 3 & -ve & Rods & $+\mathrm{ve}$ & -ve & -ve & $+\mathrm{ve}$ & -ve & -ve & K. pneumonia \\
\hline & & + ve & Rods & + ve & + ve & -ve & + ve & -ve & -ve & Staph. aurues \\
\hline \multirow[t]{4}{*}{ NNT } & 1 & $+\mathrm{ve}$ & Rods & + ve & -ve & -ve & +ve & -ve & + ve & Bacillu cereus \\
\hline & 2 & -ve & Rods & + ve & -ve & -ve & + ve & -ve & -ve & K. pneumonia \\
\hline & 3 & -ve & Rods & $+\mathrm{ve}$ & -ve & -ve & $+\mathrm{ve}$ & -ve & -ve & K. pneumonia \\
\hline & & -ve & Rods & $+\mathrm{ve}$ & -ve & -ve & $+\mathrm{ve}$ & +ve & $-\mathrm{ve}$ & P.aeruginosa \\
\hline \multirow[t]{2}{*}{ NST } & 1 & $+\mathrm{ve}$ & Rods & $+\mathrm{ve}$ & -ve & -ve & $+\mathrm{ve}$ & $-\mathrm{ve}$ & $+\mathrm{ve}$ & Bacillus cereus \\
\hline & & -ve & Rods & +ve & -ve & $+\mathrm{ve}$ & -ve & -ve & -ve & E.coli \\
\hline
\end{tabular}




\begin{tabular}{|c|c|c|c|c|c|c|c|c|c|c|}
\hline \multirow{10}{*}{ IST } & & +ve & Rods & $+\mathrm{ve}$ & + ve & -ve & $+v e$ & -ve & -ve & Staph. aurues \\
\hline & \multirow[t]{2}{*}{2} & -ve & Rods & $+\mathrm{ve}$ & -ve & -ve & $+\mathrm{ve}$ & -ve & - ve & K. pneumonia \\
\hline & & & Rods & $+\mathrm{ve}$ & +ve & -ve & +ve & -ve & -ve & Staph. aureus \\
\hline & \multirow[t]{2}{*}{3} & $+v e$ & Rods & $+\mathrm{ve}$ & -ve & -ve & $+\mathrm{ve}$ & -ve & $+v e$ & Bacillus cereus \\
\hline & & -ve & Rods & $+v e$ & -ve & -ve & $+v e$ & -ve & -ve & K. pneumonia \\
\hline & \multirow[t]{2}{*}{1} & +ve & Rods & $+\mathrm{ve}$ & -ve & -ve & $+\mathrm{ve}$ & -ve & +ve & Bacillus cereus \\
\hline & & -ve & Rods & $+\mathrm{ve}$ & -ve & $+\mathrm{ve}$ & -ve & -ve & $-\mathrm{ve}$ & E.coli \\
\hline & \multirow[t]{3}{*}{2} & $+v e$ & Rods & $+\mathrm{ve}$ & -ve & -ve & $+v e$ & -ve & $+\mathrm{ve}$ & Bacillus cereus \\
\hline & & -ve & Rods & $+\mathrm{ve}$ & -ve & -ve & +ve & +ve & -ve & P.aeruginosa \\
\hline & & -ve & Rods & $+\mathrm{ve}$ & -ve & -ve & $+\mathrm{ve}$ & -ve & -ve & K. pneumonia \\
\hline
\end{tabular}

Key: AS = Awka south, AN = Aniocha NNT = Nnewi north, NST= Nnewi south, IST = Idemili south, EKW= Ekwusigo, IH= Ihiala, NJK = Njikoka, OGB = Ogbaru, ONT = Onitsha nouth, OST= Onitsha south

Tables 4.8: B) Biochemical And Characterization Of Bacterial Isolates From Suya Meat

\begin{tabular}{|c|c|c|c|c|c|c|c|c|c|c|}
\hline $\begin{array}{l}\text { Sample } \\
\text { site }\end{array}$ & $\begin{array}{l}\text { Sample } \\
\text { code }\end{array}$ & $\begin{array}{l}\text { Grams } \\
\text { reaction }\end{array}$ & $\begin{array}{l}\text { Cellular mor- } \\
\text { phology }\end{array}$ & Catalase & Coagulase & Indole & Citrate & Oxidase & $\begin{array}{l}\text { Starch hydrol- } \\
\text { ysis }\end{array}$ & $\begin{array}{l}\text { Probable } \\
\text { identity }\end{array}$ \\
\hline \multirow{6}{*}{ EKW } & 3 & $+\mathrm{ve}$ & Rods & +ve & -ve & -ve & + ve & -ve & $+\mathrm{ve}$ & $\begin{array}{l}\text { Bacillus cere- } \\
\text { us }\end{array}$ \\
\hline & \multirow{4}{*}{1} & -ve & Rods & $+v e$ & -ve & -ve & + ve & -ve & -ve & K. pneumonia \\
\hline & & $+\mathrm{ve}$ & Rods & $+\mathrm{ve}$ & -ve & -ve & $+\mathrm{ve}$ & -ve & $+\mathrm{ve}$ & $\begin{array}{l}\text { Bacillus cere- } \\
\text { us }\end{array}$ \\
\hline & & $+\mathrm{ve}$ & Rods & $+\mathrm{ve}$ & $+\mathrm{ve}$ & -ve & + ve & -ve & -ve & Staph. Aureus \\
\hline & & -ve & Rods & $+\mathrm{ve}$ & -ve & -ve & + ve & -ve & -ve & K. pneumonia \\
\hline & 2 & +ve & Rods & +ve & -ve & -ve & +ve & -ve & +ve & $\begin{array}{l}\text { Bacillus cere- } \\
\text { us }\end{array}$ \\
\hline \multirow{9}{*}{$\mathrm{IH}$} & \multirow{3}{*}{3} & $+\mathrm{ve}$ & & $+\mathrm{ve}$ & $+\mathrm{ve}$ & -ve & $+\mathrm{ve}$ & -ve & -ve & Staph. Aureus \\
\hline & & \multirow[t]{2}{*}{$+\mathrm{ve}$} & Rods & +ve & -ve & -ve & + ve & -ve & $+\mathrm{ve}$ & $\begin{array}{l}\text { Bacillus cere- } \\
\text { us }\end{array}$ \\
\hline & & & Rods & $+\mathrm{ve}$ & -ve & $+\mathrm{ve}$ & -ve & -ve & -ve & E.coli \\
\hline & \multirow{3}{*}{1} & + ve & Rods & $+v e$ & $+\mathrm{ve}$ & - ve & $+\mathrm{ve}$ & -ve & -ve & Staph. Aureus \\
\hline & & + ve & Rods & $+\mathrm{ve}$ & -ve & -ve & + ve & -ve & $+\mathrm{ve}$ & $\begin{array}{l}\text { Bacillus cere- } \\
\text { us }\end{array}$ \\
\hline & & -ve & Rods & $+\mathrm{ve}$ & -ve & -ve & $+\mathrm{ve}$ & -ve & -ve & E.coli \\
\hline & \multirow[t]{2}{*}{2} & $+v e$ & Rods & $+\mathrm{ve}$ & -ve & -ve & $+\mathrm{ve}$ & -ve & $+\mathrm{ve}$ & $\begin{array}{l}\text { Bacillus cere- } \\
\text { us }\end{array}$ \\
\hline & & $+\mathrm{ve}$ & Rods & $+\mathrm{ve}$ & $+\mathrm{ve}$ & -ve & $+\mathrm{ve}$ & -ve & -ve & Staph. Aureus \\
\hline & \multirow[t]{4}{*}{3} & $+\mathrm{ve}$ & Rods & +ve & -ve & -ve & $+\mathrm{ve}$ & -ve & $+\mathrm{ve}$ & $\begin{array}{l}\text { Bacillus cere- } \\
\text { us }\end{array}$ \\
\hline \multirow{7}{*}{ NJK } & & $+v e$ & Rods & $+\mathrm{ve}$ & $+v e$ & -ve & $+v e$ & $-\mathrm{ve}$ & -ve & Staph. Aureus \\
\hline & & -ve & Rods & $+\mathrm{ve}$ & -ve & - ve & $+v e$ & $-\mathrm{ve}$ & -ve & K. pneumonia \\
\hline & & -ve & Rods & $+\mathrm{ve}$ & -ve & -ve & -ve & $-\mathrm{ve}$ & -ve & Salmonella \\
\hline & 1 & $+\mathrm{ve}$ & Rods & $+\mathrm{ve}$ & $+\mathrm{ve}$ & -ve & $+\mathrm{ve}$ & -ve & -ve & Staph. Aureus \\
\hline & 2 & $+\mathrm{ve}$ & Rods & $+\mathrm{ve}$ & -ve & -ve & $+\mathrm{ve}$ & -ve & $+\mathrm{ve}$ & $\begin{array}{l}\text { Bacillus cere- } \\
\text { us }\end{array}$ \\
\hline & & $+\mathrm{ve}$ & Rods & $+v e$ & + ve & -ve & + ve & -ve & -ve & Staph. Aureus \\
\hline & & -ve & Rods & -ve & -ve & -ve & $+\mathrm{ve}$ & -ve & -ve & K. pneumonia \\
\hline \multirow{3}{*}{ OGB } & 3 & $+\mathrm{ve}$ & Rods & $+v e$ & $+\mathrm{ve}$ & -ve & $+\mathrm{ve}$ & -ve & -ve & Staph. Aureus \\
\hline & \multirow[t]{2}{*}{1} & \multirow[t]{2}{*}{$+\mathrm{ve}$} & Rods & $+v e$ & -ve & -ve & $+v e$ & $-\mathrm{ve}$ & $+\mathrm{ve}$ & $\begin{array}{l}\text { Bacillus cere- } \\
\text { us }\end{array}$ \\
\hline & & & Rods & $+\mathrm{ve}$ & -ve & $+\mathrm{ve}$ & -ve & -ve & -ve & E.coli \\
\hline
\end{tabular}

Key: AS = Awka south, AN = Aniocha NNT = Nnewi north, NST= Nnewi south, IST = Idemili south, EKW= Ekwusigo, IH= Ihiala, NJK = Njikoka, OGB $=$ Ogbaru, ONT $=$ Onitsha nouth, OST= Ontisha south.

Tables 4.8: C) Biochemical and Characterization Of Bacterial Isolates From Suya Meat

\begin{tabular}{|c|c|c|c|c|c|c|c|c|c|c|}
\hline $\begin{array}{l}\text { Sample } \\
\text { site }\end{array}$ & $\begin{array}{l}\text { Sample } \\
\text { code }\end{array}$ & $\begin{array}{l}\text { Grams } \\
\text { reaction }\end{array}$ & $\begin{array}{l}\text { Cellular mor- } \\
\text { phology }\end{array}$ & Catalase & Coagulase & Indole & Citrate & Oxidase & $\begin{array}{l}\text { Starch hydrol- } \\
\text { ysis }\end{array}$ & $\begin{array}{l}\text { Probable } \\
\text { identity }\end{array}$ \\
\hline & 2 & -ve & Rods & +ve & -ve & -ve & $+\mathrm{ve}$ & -ve & $+v e$ & $\begin{array}{l}\text { Bacillus cere- } \\
\text { us }\end{array}$ \\
\hline & & +ve & Rods & $+v e$ & $+\mathrm{ve}$ & $-v e$ & $+\mathrm{ve}$ & -ve & -ve & Staph. Aureus \\
\hline & & -ve & Rods & +ve & -ve & +ve & -ve & -ve & -ve & E.coli \\
\hline & 3 & $+v e$ & Rods & +ve & -ve & -ve & $+\mathrm{ve}$ & -ve & $+\mathrm{ve}$ & $\begin{array}{l}\text { Bacillus cere- } \\
\text { us }\end{array}$ \\
\hline & & $+v e$ & Rods & $+v e$ & $+\mathrm{ve}$ & -ve & +ve & -ve & -ve & Staph. Aureus \\
\hline & & -ve & Rods & +ve & -ve & -ve & -ve & -ve & -ve & Salmonella \\
\hline & & -ve & Rods & + ve & -ve & + ve & -ve & $-\mathrm{ve}$ & -ve & E.coli \\
\hline \multirow[t]{6}{*}{ OST } & 1 & -ve & Rods & +ve & -ve & -ve & -ve & -ve & $-v e$ & Salmonella \\
\hline & & $+\mathrm{ve}$ & Rods & $+\mathrm{ve}$ & $+\mathrm{ve}$ & -ve & $+\mathrm{ve}$ & -ve & $-v e$ & Staph. Aureus \\
\hline & & $-\mathrm{ve}$ & Rods & + ve & -ve & -ve & $+v e$ & $+v e$ & -ve & P. aeruginosa \\
\hline & 2 & $+\mathrm{ve}$ & Rods & +ve & -ve & -ve & $+\mathrm{ve}$ & -ve & $+\mathrm{ve}$ & $\begin{array}{l}\text { Bacillus cere- } \\
\text { us }\end{array}$ \\
\hline & & -ve & Rods & +ve & -ve & -ve & -ve & -ve & $-v e$ & Staph. Aureus \\
\hline & 3 & -ve & Rods & + ve & -ve & $-v e$ & $+\mathrm{ve}$ & $-\mathrm{ve}$ & $-\mathrm{ve}$ & K. pneumonia \\
\hline \multirow[t]{4}{*}{ ONT } & 1 & -ve & Rods & $+\mathrm{ve}$ & -ve & -ve & $+\mathrm{ve}$ & -ve & $-v e$ & K. pneumonia \\
\hline & 2 & -ve & Rods & $+\mathrm{ve}$ & -ve & -ve & -ve & -ve & $-v e$ & Salmonella \\
\hline & & -ve & Rods & + ve & -ve & -ve & $+v e$ & $-\mathrm{ve}$ & $-\mathrm{ve}$ & K. pneumonia \\
\hline & 3 & -ve & Rods & +ve & -ve & -ve & -ve & -ve & -ve & Salmonella \\
\hline OYI & 1 & -ve & Rods & +ve & -ve & -ve & $+\mathrm{ve}$ & -ve & -ve & K. pneumonia \\
\hline
\end{tabular}




\begin{tabular}{|c|c|c|c|c|c|c|c|c|c|c|}
\hline & 2 & $+\mathrm{ve}$ & Rods & $+\mathrm{ve}$ & -ve & -ve & $+\mathrm{ve}$ & -ve & $+\mathrm{ve}$ & $\begin{array}{l}\text { Bacillus cere- } \\
\text { us }\end{array}$ \\
\hline & 3 & -ve & Rods & $+\mathrm{ve}$ & -ve & -ve & -ve & -ve & -ve & Salmonella \\
\hline & & & Rods & $+\mathrm{ve}$ & -ve & +ve & -ve & -ve & -ve & E.coli \\
\hline Control & & -ve & - & -ve & -ve & -ve & -ve & -ve & -ve & ND \\
\hline
\end{tabular}

Key: AS = Awka south, AN = Aniocha NNT = Nnewi north, NST= Nnewi south, IST = Idemili south, EKW= Ekwusigo, IH= Ihiala, NJK = Njikoka, OGB = Ogbaru, OST $=$ Onitsha south, ONT= Onitsha

\section{Discussion}

The levels of some selected heavy metals (cadmium, lead, zinc and arsenic) and microbial loads were analyzed in fresh suya samples in different senatorial zones in Anambra state. Heavy metal levels and microbial loads in suya varied in the different zones in the state. Suya is a rich source of essential nutrients for humans. Its nutritional contamination, characteristics and how it was processed makes an avenue for possible heavy metals deposition and an excellent medium for microbial proliferation leading to rapid deterioration, loss of organoleptic property and economy (Orogu et al., 2016). This phenomenon may be attributed to poor personal hygiene and water quality, uncovered suya, traditional processing techniques and unhealthy environment.

Results from the analysis confirmed the presence of cadmium, lead, zinc and arsenic in all of the selected suya samples in all the zones. The highest concentration of cadmium was found in Anambra south while that of lead was found in Anambra north. Furthermore, the highest value of zinc and arsenic was found in Anambra north. Lead is classified as one of the most toxic heavy metals with no biochemical benefits to humans and animals (Akoto et al., 2014). The wide spread industrial production of perfumes, batteries, cement production, quarrying especially limestone and brick making as well as agricultural discharges, sewage effluents, high way or vehicular traffic are considered main sources of lead (Isa et al., 2015). Lead detected in $63 \%$ of the suya samples in all the three zones were above the maximum permissible limits of lead set by FAO/WHO $(0.2 \mathrm{mg} / \mathrm{kg})(\mathrm{FAO} / \mathrm{WHO}, 2011)$ and Codex Alimentorum $(0.4 \mathrm{mg} / \mathrm{kg})(\mathrm{CODEX}$, 2011) in meat.

The excess amount of lead in the samples may be attributed to contamination during production as suya is usually displayed in the open which makes it susceptible to automobile emission. Lead concentration in this study was higher than those reported by Dibofori-Orji and ThankGod (2018). Fedrick et al (2015) reported mean lead concentration in grilled meat as $0.63 \mathrm{mg} / \mathrm{kg}$ to $1.63 \mathrm{mg} / \mathrm{kg}$. It is suggested that the spices used to make suya meat could be a possible cause for this excess concentration and the place in which the coal was obtained might also have a contribution to the increased lead $(\mathrm{Pb})$ level. A high level of lead in adult's body can predispose to heart diseases, cancer and infertility. For children, the diseases caused by lead can result in antisocial behaviour, low intelligence or hyperactivity (Canfield et al., 2003).

Cadmium is a toxic element to every animal species and for humans, as well. It is almost absent in the human and animal body at birth, however it accumulates with age. In the current study, cadmium was detected in all the suya samples with $100 \%$ of the suya sample seen to be higher than the maximum permissible limit set by WHO ( $0.5 \mathrm{mg} / \mathrm{kg})($ FAO/WHO, 2011) and CODEX (0.1mg/kg) (CODEX, 2011) in meat. The high level of cadmium observed in this study is similar to $5.46 \mathrm{mg} / \mathrm{kg}$ reported by Dibofori-Orji and ThankGod(2018). Cadmium is an environmental contaminant unique among metals because of its diverse toxic effects, extremely protracted biological half life, low rate of excretion from the body and predominant storage in soft tissues (Benova et al., 2007). Cadmium in food is the most important source of cadmium exposure to non- smoking population in most countries (WHO, 2002). Cadmium is present in insecticides, fungicides, sludge and fertilizers used in agriculture. Organs such as the liver, placenta, kidneys, lungs, brain and bones can be affected by cadmium exposure (Alissa and Fern 2011). The high level of cadmium and lead in the suya samples may be attributed to the roasting process since wood ashes contain heavy metals which may penetrate the suya meat (Garcia et al., (2004). Also, cadmium contamination of the suya meat may emanate from emissions due to disposal and burning of hazardous wastes such as electronics, used vehicle tyres and nickel cadmium batteries.

The mean concentration of zinc obtained in suya samples was observed to be in the range of 2.6 to $27.3 \mathrm{mg} / \mathrm{kg}$. Federick et al (2015) found $\mathrm{Zn}$ concentrations between $1.45-2.24 \mathrm{mg} / \mathrm{kg}$ in grilled beef and chicken. In this study, higher concentrations of $\mathrm{Zn}$ were observed but below the guideline value of $60 \mathrm{mg} / \mathrm{kg}$ set by CODEX in meat (Codex, 2011). This finding suggests that zinc contamination does not take place in the environment where the samples were collected and also where the animals feed Arsenic was also detected in the suya meat sample with $89 \%$ seen to be above the permissible limit set by WHO $(0.1 \mathrm{mg} / \mathrm{kg})(\mathrm{FAO} / \mathrm{WHO}, 2011)$ in all the zones in Anambra state. The result obtained in this study was higher than the arsenic content $(0.3 \mathrm{mg} / \mathrm{kg}$ ) found in beef in Maiduguri (Akan et al., 2010), this could be attributable to the method of preparation. Arsenic is a highly toxic metal that affects nearly all organs of the body. It is strongly associated with lung and skin cancers and may cause renal failure, peripheral vascular changes, gangrene of the extremities, peripheral neuropathy, hyper-pigmentation, hyperkeratosis, bone marrow depression, spontaneous abortion and congenital malformation (Appiah, 2016).

The locations where these samples were collected also contributed to the high level of heavy metal contamination because of the activities carried out in such environments. Anambra state is a highly polluted area with different industries which carry out activities such as production of automobiles and plastics, automobile lead acid- acid battery and spare parts, production of fertilizers, metallurgical works, among others, without the necessary safety precautions. These activities can pollute the air and suya being usually uncovered; there is a possibility that it can be contaminated by the contaminants. The highest concentration of metals especially cadmium and lead were found in Anambra south and north. This observation could be as a result of high industrial activities in the southern part of the state and high urbanization in Anambra North.

In the control sample, it was observed that the heavy metals analyzed (cadmium, lead, zinc and arsenic) were detected but the values were low compared to the test samples analyzed. These values of heavy metals detected could be attributed to the fact that cattle feed on plants and water which are contaminated by these heavy metals. Okoye and Ibeto (2008) reported high levels of lead and cadmium in soils from Enugu state which could be transferred to plants and this could serve as a source of heavy metal contamination in animals grazing in such area of the state likewise in Anambra state and other locations. Indeed, close correlation have been reported between heavy metals concentration in cattle tissues with that in soil, feed, and drinking water (Qiu et al., 2008). The control sample was prepared in the laboratory free from heavy metal emission using an oven with grill instead of the wood ash and coal used majorly by the suya sellers. This could also contribute to the low level of heavy metals observed in comparison with the samples collected from different zones in Anambra state. The estimated daily intake values of lead, arsenic and zinc for all the zones were lower than the provisional maximum tolerable daily intake (PMTDI) of lead $(0.002-3 \mu \mathrm{g} / \mathrm{kg} /$ day), arsenic $(2.1 \mu \mathrm{g} / \mathrm{kg} /$ day) and zinc $(1000 \mu \mathrm{g} / \mathrm{kg} / \mathrm{day})(\mathrm{FAO} / \mathrm{WHO}, 2012)$. 
The EDI values of cadmium for all the zones were higher than the PMTDI of cadmium $(0.8 \mu \mathrm{g} / \mathrm{kg} / \mathrm{day})$ (FAO/WHO, 2012). This observation is noteworthy and of significant public health importance because daily intake of suya may likely cause significant hazard to the residents. The estimated daily intake of heavy metals is an important exposure pathway in assessment of risk (Rahman et al., 2014).

Target hazard quotient (THQ) values of the metals in this study present public concern with THQ > 1 in the suya sample. The THQ of cadmium was greater than 1 in all the zones in Anambra state. These values indicate that suya meat is unsafe for human consumption. The THQ for lead, zinc and arsenic can be considered to be safe for consumers due to their low non carcinogenic risk presenting values < 1. Hazard Index (HI) is the total of all the THQ values in food samples and a value $>1$ indicates that the probability of an adverse health effect associated with such exposure is high. The HI values were greater than 1 in all the zones in Anambra state. The hazard index indicates that suya processed in different zones pose risks to consumers due to accumulation of heavy metal contaminants with values greater than one. The highest hazard index value however was found in suya sample from Anambra south. This demonstrates that consumption of suya in different zones in Anambra state pose a potentially great non-carcinogenic risk.

Cancer risk of an individual is estimated by calculating the increasing chances to develop cancer over a life time due to exposure to a contaminant. For example, a cancer risk of $1 \times 10^{-4}$ indicates a probability of 1 in 10,000 individuals developing cancer. Among the four elements assayed, lead, arsenic and cadmium were of greatest concern for their carcinogenicity (IARC, 2011). The USEPA considers acceptable for regulatory purposes a cancer risk in the range of 1.E-06 $\left(1 \times 10^{-6}\right)$ to $1 . \mathrm{E}-04\left(1 \times 10^{-4}\right)$ (USEPA, 2011). This means that any value greater than $10^{-4}$ is unacceptable risk. In this study, the cancer risk for arsenic (9.00E-3) in samples from Anambra north was more than the acceptable limit while other locations were within the acceptable limit. Furthermore, cadmium had cancer risk above the acceptable limit in all the zones. This suggests that for the toxic metal cadmium, the carcinogenic risk through the consumption of suya meat should be of great concern. Long-term environmental exposure to cadmium has been reported to be associated with an increased risk of all-cancer mortality (Wang et al., 2011). A study in the European countries suggested correlations between cadmium level and the age-adjusted prostate or breast cancer rates (Pan et al., 2010). The cancer risk for lead for all the groups falls within the USEPA acceptable limit.

Microbiological analysis was carried out on suya meat collected randomly in the different zones in Anambra state, show that the total plate counts in suya samples analyzed are similar to earlier reports (Hassan et al., (2014) on suya where the total viable count obtained ranged from $9.4 \times 10^{6} \mathrm{cfu} / \mathrm{g}$ to $7.65 \times 10^{6} \mathrm{cfu} / \mathrm{g}$. This study is also in agreement with the reports by Osakue et al (2016) on street vended fried chicken in Benin City, Nigeria with a total viable count which ranged from $5.8 \times 10^{6} \mathrm{cfu} / \mathrm{g}$ to $1.12 \times 10^{7} \mathrm{cfu} / \mathrm{g}$. The total viable count obtained from Nnewi north and south, Idemili, Ekwusigo, Ihiala, Ogbaru Onitsha north and south which were too numerous to count (TNTC) are greater than the permissible limit $<10^{5}$ set by Microbiological Guidelines for ready to eat food (Gilbert, 2000) and the consumption of suya meat greater than the permissible limit could result to some health problems like food poisoning and intoxication. The presence of fungi in food product is undesirable (Adegoke, 2004) and have been implicated in food poisoning illness and, are also known as spoilage microorganisms. The total fungi count obtained from this study is low. Six organisms were isolated from the suya samples in the present study and included S. aureus, B. cereus, Samonella spp,P. aeruginosa, K. pneumonia and E. coli. This is in agreement with earlier studies which isolated E. coli, S. aureus, B. cereus, K. aerogenes, P. aeruginosa from suya meat sold in Awka Anambra State (Onuora et al., 2015) and B. subtilis, E. coli and S.aureus from meat sold in Ozoro, Delta State. In view of the unhygienic conditions prevalent with meat handling in Nigeria, these isolated organisms may be linked with cases of meat contamination and spoilage.

The presence of Staphylococcus aureus affirms the cross contamination through processing (Gilbert and Harisson, 2001) which is in agreement with the reported high incidence of coagulase positive Staphylococcus auerus and E. coli in Kilishi from Kano metropolis (Shamsuddeen et al., 2008). The presence of Staphylococcus aureus in the suya meat reveals that contamination can be from the hands of the sellers since it is commonly found in hands, skin, utensils and air (Bukar et al., 2009) and even the ingredients like the spices which have been implicated as a source of contamination (Frazier et al., 2006). It could also be attributed to the raw sliced onions, cabbage and poor environment in which the suya is prepared. Staphylococcus aureus is an opportunistic pathogen and the enterotoxigenic strains of it are known to cause food born disease (Prescott et al., 2005). The meat also showed presence of Pseudomonas aeruginosa which is widely spread in nature especially in soil, water and plants (Field, 2002) and can easily contaminate suya sample usually displayed uncovered to attract customers. The presence of E. coli and Klebsiella pneumonia calls for concern as these organisms are frequently associated with poor sanitary practices and could be a pointer to the danger of possible food- borne infections. E. coli is an indicator of poor hygiene and fecal pollution. Bacillus cereus is a spore former. It can be found in the air and even spices. The spores are heat resistant and this may account for its occurrence in suya. B. cereus was isolated from all the suya samples and the presence of Bacillus species rendered the samples unsatisfactory (PHLS, 2000).

Several reports have implicated S. aureus and Bacillus cereus to cause food borne diseases due to their ability to produce thermo-stable toxins and spores respectively (Mensah et al., 2012; Okwori et al., 2014). Additionally, health conditions may be exacerbated by the ability of E. coli, S. aureus, S. epidermidis and K. pneumonae to form biofilms which enhances antibiotic resistance (Chen et al., 2013). Reports also reveal that an estimated $40-50 \%$ of prosthetic valve infections and $50-70 \%$ catheter biofilm infections as well as $87 \%$ of blood stream infections are attributed to Staphylococci (Agarwal et al., 2010; Chen et al., 2013). The suya processors as observed were all middle-aged Hausa Fulani illiterate men without any kind of formal education. None of them had any training in food preparation which is necessary and important for hygienic handling of foods especially for a nutritious food like suya made from a substrate that is also an excellent culture medium for a wide variety of microorganisms. Personal hygiene of suya processors was observed; they all used bare hands to handle both food and money simultaneously. Personal hygiene is imperative because humans are the largest sources of contamination in food and any food handler who observes other forms of hygiene but not personal hygiene will definitely contaminate food. All suya processors prepared the suya at their wooden stalls located by the roadsides. The surroundings were considered unhygienic given that garbage and dirty wastes littered around the food processing environment with open gutters nearby, all of which attracted houseflies.

Slabs and trays used for cutting and sticking were inadequately cleaned. Prepared suya samples were not covered but left exposed to flies and dust during display. They were kept at ambient temperature and the re-heating temperature of less than $70{ }^{\circ} \mathrm{C}$ was not sufficient to destroy pathogens. The roasting period for suya is on the average 30 minutes at about $70{ }^{\circ} \mathrm{C}$. This time and temperature regime may not be sufficient to destroy all the vegetative cells and heat-resistant spores of bacteria especially if the meat is heavily contaminated with enteric bacteria (Chen et al., 2013). Heat treatment of food such as roasting not only improves the taste, smell, appearance and digestibility of the food, it also reduces the number of microorganisms, improves keeping qualities by inhibiting moulds, yeast and bacteria that promote decay and infection. Thus, heat treatment is a practice aimed at improving the overall safety of food. In many cases suya is not consumed immediately after preparation. It is held at ambient temperature for more than 5 hours before serving. The temperature of the meat after reheating is about $50{ }^{\circ} \mathrm{C}$. This time and temperature regime was not adequate for destroying all vegetative bacterial cells $(\mathrm{Chen}$ 
et al., 2013). Holding suya at ambient temperature for too long could be risky since this could encourage the growth of the pathogens to hazardous levels.

No growth was found in the control sample because all the hygienic processes were judiciously followed.

\section{Conclusion}

This study has shown that suya is prepared and sold under largely unhygienic and un-safe conditions in the studied location of Anambra state thereby constituting a food safety risk, in this case microbiological and heavy metals contamination, to the numerous and everincreasing consumers.

\section{References}

[1] Akoto, O., Bismark,F., Darko, G. and Adei, E. (2014).Concentrations and Health Risk Assessment of Heavy Metals in Fish from the Fosu Lagoon. International Journal of Environmental Research, 8(2): 403-410.

[2] Akpamu, N.I. (2011). A comparative study on the acute and chronic effect of oral administration of Yaji (A complex Nigerian Meat Sauce) on Soue Hematological Parameters.British Journal of Pharmacology and Toxicology 3(2):108-112.

[3] Alissa, E.M. and Fern, G.A. (2011). Heavy metal poisoning and cardiovascular disease .Journal of Toxicology, 870125: 1-21. https://doi.org/10.1155/2011/870125.

[4] Appiah, A.I. (2016). Assessment of microbial quality and heavy metal levels of raw cattle hide and meat sold at retail outlets in Tarkwa, Western region, Ghana.M.Sc Thesis; Kwame Nkrumah University of Science and Technology, Kumasi.

[5] Benova, K., Dvorak, P., Falis, M. and Sklenar, Z. (2007). Interaction of low doses of ionizing radiation potassium dichromate and cadmium chloride in Artemia franciscama.ActaVeterinaria Brno. 76:35-40. https://doi.org/10.2754/avb200776010035.

[6] Beyersmann, D. and Hawtwig, A. (2008). Carcinogenic metal compounds: recent insight into molecular and cellular mechanisms. Archive of Toxicology. 82(8): 493-512. https://doi.org/10.1007/s00204-008-0313-y.

[7] Caulfield, L.E, Zavaleta, N., Shenkar, A.H. and Merialdi, M. (2000).Potential contribution of maternal zinc supplement during pregnancy to maternal and child survival. The American Journal of Clinical Nutrition, 68:499-508. https://doi.org/10.1093/ajcn/68.2.499S.

[8] Cheesbrough, M. (2009).District Laboratory Practice in Tropical Countries. Cambridge university UK, 2(2): 65-67.

[9] Chukwuma, E.C. and Orakwe, L.C.(2017). An application of facility location models with hotspot analysis for optimal location of abattoir bio energy plant in Anambra state of Nigeria . International Journal of science and Technology Research, vol. 5(4) 173-179.

[10] Codex Alimentarius Commission.(2011). Joint FAO/WHO Food Standards Programme, Codex committee on Contaminants in Foods. Fifth Season, the Hague, the Netherlands, 21-25 March 2011.

[11] Dibofori-Orji, A.N. and ThankGod, P. (2018).Analysis of some heavy metals in hawked charcoal roasted beef (suya) within Port Harcourt metropolis .European Journal of Pure and Applied Chemistry, 5:12-19.

[12] Egbebi, A.O. and Seidu, K.T. (2011). Microbiological evaluation of Suya (dried smoked meat) sold in Ado and Akure South West Nigeria. European Journal of Experimental Biology, 1(4):1-5.

[13] Eke, S.O., Irabor, J.I., Okoye, M., Aitufe, O.F. and Ekoh, S.N. (2013). The microbial status of commercial 'Suya' meat products in Ekpoma, Edo, Nigeria.International Journal of Community Resources, 2(1):18-21.

[14] FAO/WHO. (2011). Cadmium, In: Safety Evaluation of Certain Food Additives and Contaminants, Fifty-Fifth Meeting of the Joint FAO/WHO Expert Committee on Food Additives (JECFA), Geneva, World Health Organization Food Additive Series, 273-312.

[15] Frederick, A., Andrew, K. and Seddoh, B. (2015). Assessment of the presence of selected heavy metals and their concentration levels in fresh and grilled beef/ guinea fowl meat in the Tamala Metropolis, Ghana. Resarch Journal of Environmental Sciences, 9(3):152-158. https://doi.org/10.3923/rjes.2015.152.158.

[16] Garcia, M.S., Perez, C. and Simal-Gandara, J.(2004). Strategies for the extraction of free and bound polycyclic aromatic hydrocarbons in wood ash used in fertilizer in agroforestry soils and their transfer from ashes to water.Journal for agricultural and Food Chemistry, 508(2): 177-183).

[17] Hărmanescu, M., Alda, L.M., Bordean, D.M., Gogoaşă, I. and Gergen, I. (2011). Heavy metals health risk assessment for population via consumption of vegetables grown in old mining area; a case study: Banat County, Romania, Chemistry Central Journal, 5. https://doi.org/10.1186/1752 $153 \mathrm{X}-5-64$.

[18] IARC. (2011). Oxford Handbook of Occupational Health; Agents Classified by the IARC Monographs. OUP Oxford: Oxford, UK. Orogu, J.O., Oshilim, A.O. ( 2016). Comparative study of bacteriological analysis in hawked suya meat and suya meat on barbeque stand.Journal of Microbiology Research 3(1):005-008. https://doi.org/10.18685/EJMBR(3)1 EJSR-16-025.

[19] Innocent, O., Agbowu, B. and Agbo, F. (2017). Proximate composition , microbiological safety and heavy metal contamination of garri sold in Benue, North -Central, Nigeria. African Journal of Biotechnology. 18: 1085-1091). https://doi.org/10.5897/AJB2016.15841.

[20] Inyang, C.U., Igyor, M.A. and Uma, E.N. (2005).Bacterial Quality of a Smoked Meat product (Suya).Nigerian Food Journal, 23: 239-242. https://doi.org/10.4314/nifoj.v23i1.33622.

[21] Isa, B.K., Amina, S.B., Aminu, U. and Sabo, Y. (2015).Health risk assessment of heavy metals in water, air, soil and fish.African Journal of Pure and Applied Chemistry, 9(11): 1-7. https://doi.org/10.5897/AJPAC2015.0654.

[22] Lansdown, A.B., Mirastschijski, U., Stubbs, N., Scanlon, E. and Agren, M.S.( 2007). Zinc in wound healing: theoretical, experimental and cinical aspects. Wound Repair Regen; 15: 2-16. https://doi.org/10.1111/j.1524-475X.2006.00179.x.

[23] Mead, G.C. (2004). Microbiological quality of poultry meat: A review. Brazilian Journal of Poultry Science, 6:135-142. https://doi.org/10.1590/S1516-635X2004000300001.

[24] Moshood, A.Y. and Tengku H., Ibrahim, H. (2012). Isolation and Identification of Bacteria Associated with Balangu (roasted meat product) sold in Bauchi, Nigeria Journal of Pharmacy, 2(6):38-48. https://doi.org/10.9790/3013-26503848.

[25] Odey, M.O., Mboso, E.O., Ujong, U.P., Johnson, J.T., Gauje, B. and Ategwu, M.A. (2013).Microflora analysis of selected meat and meat products from Calabar, Cross Rivers State Nigeria.Scholars Research Library Archives of Applied Science Research, 5(3): 50-56.

[26] Ojebah, C.K. and Ewhre, O. L (2015). Heavy metal contamination in suya meat marketed in selected towns in Delta State. Journal of environmental science, pp 110-113.

[27] Okoye, C.O.B. and Ibeto, C.N. (2008).Book of Proceedings of the $31^{\text {th }}$ Annual International Conference of the Chemical Society of Nigeria. Warri pp $767-771$.

[28] Omojola, A.B. (2008). Yield and organoleptic characteristics of suya (an intermediate moisture meat) prepared from three different muscles of a matured bull. African Journal of Biotechnology, 7(13), 2254-2257.

[29] Pavydê, E., Veikutis, V., Mačiuliene, A., Mačiulis, V., Petrikonis, K. and Stankevičius, E. (2015). Public Knowledge, Beliefs and behavior on antibiotic Use and Self-Medication in Luthuania.International Journal of Environmental Research and Public Health, 12:7002-7016. https://doi.org/10.3390/ijerph120607002.

[30] Potts, P., Ellis, A., Marshall, J. and Streli, C. (2004). Concepts, Instrumentation and Techniques in Atomic Absorption Spectrophometry, The Perkin-Elmer Corporation ( $2^{\text {nd }}$ Edition $)$.

[31] Qiu, C.A.I., Long, M., Liu, J., Zhu, M., Zhou, Q., Deng, Y., Li. and Tain, Y.J. (2008). Correlation between heavy metals concentration in cattle tissues and rearing environment.Chinese Journal of Ecology 27(02): 202-207. 
[32] Reiner, K. (2010). Catalase test protocol.American Society for Microbiology, ASM Microbe Library.

[33] Salihu, M.D., Junaidu, A.U., Magaji, A.A., Aliyu, R.M., Yakubu, Y., Shittu, A., and Ibrahim, M.A. (2013).Bacteriological quality of traditionally prepared fried ground Beef (Dambunnama) in Sokoto, Nigeria.Advance Journal of Food Science Technology, 2(3):145-147.

[34] United States Environmental Protection Agency. (2012). Waste and cleanup risk assessment. Available online http://www2.epa.gov/risk/wasteand-cleanup-risk-assessment [Assessed on $20^{\text {th }}$ October 2017].

[35] Uzeh, R.E., Ohenhen, R.E. and Adeniji, O.O (2006).Bacterial contamination of Tsire-Suya, a Nigerian Meat Product.Pakistian Journal of Nutrition, 5(5):458-460. https://doi.org/10.3923/pjn.2006.458.460.

[36] Winter, D.H. (2015). Public health implications asociated with the practice of utilizing tires to singe meat in three major cities of Ghana: A concurrent mixed methods study. Available online http://digitalcommons.georgiasouthern.edu/etd. [Assessed on 12th September 2017].

[37] Wintergerst, E.S., Maggini, S. and Horning, D.H (2007). Contribution of selected vitamins and trace elements in immune function .Annals of Nutrition and Metabolism; 51: 301-302 https://doi.org/10.1159/000107673. 